\section{Brain space for SIRT1}

\section{By Lev Osherovich, Senior Writer}

A trio of mouse studies suggests that sirtuin 1 acts in brain regions that control appetite, learning and memory. ${ }^{1-3}$ The finding adds a new dimension to the protein deacetylase's known role in regulating energy sensing in the peripheral organs. In addition, the research suggests that activation of sirtuin 1 could open up neurological indications for companies already pursuing the target in the metabolic space, provided that compounds can be precisely directed to the brain.

The studies all used tissue-specific knockout mice to pinpoint the behavioral effects of sirtuin 1 (Sirt1) deficiency in the brain and found that the absence of Sirt 1 either magnified the effect of metabolic and neurodegenerative disease or blocked adaptive mechanisms that counteract disease processes. The unanswered question is whether upregulating SIRT1 will have a therapeutic effect in neurological diseases.

Prior studies have shown that whole-body Sirt1 knockout mice have problems sensing energy levels in the liver and muscles and thus are unable to adapt to excessively rich or poor diets. ${ }^{4}$ Based on those findings, independent teams at the Washington University in St. Louis and The University of Texas Southwestern Medical Center at Dallas examined the effect of brain-specific Sirt1 deletion on the behavioral and metabolic response to dietary stress.

A group led by Roberto Coppari, assistant professor of internal medicine at University of Texas Southwestern, created hypothalamusspecific Sirt 1 knockout mice and characterized their metabolism and brain activity on a high-fat diet. ${ }^{1}$

Female mice lacking the Sirt1 gene in the proopiomelanocortin (POMC) neurons of the hypothalamus showed greater fat deposition and weight gain on a high-calorie diet than wild-type controls. POMC neuron-specific Sirt1 knockouts had normal food intake but lower metabolic rates than wild-type controls.

The team went on to show that the hypothalamus of Sirt1-deficient mice does not respond correctly to leptin, an adipocyte-derived hormone that helps to signal satiety.

"POMC neurons are metabolic sensor neurons, sensing glucose and fatty acids as well as hormones. Sirt1 is required for POMC neurons to be engaged properly by leptin," Coppari told SciBX. "If you have a Sirt1 mutation in the POMC [neurons] in the hypothalamus, you're more prone to develop diabetes if you're exposed to an obesogenic environment."
Coppari's results were published in Cell Metabolism.

Whereas Coppari's group looked at mice on a high-fat diet, a team led by Shin-ichiro Imai, associate professor of developmental biology and internal medicine at Washington University in St. Louis, went in the opposite direction and tested how knocking out Sirt1 in the brain affected metabolic adaption to a low-calorie diet. ${ }^{2}$

Indeed, Imai told SciBX that his team's findings, which were published in The Journal of Neuroscience, are the flip side of Coppari's work.

Imai's group demonstrated that mice with a brain-specific deletion of Sirt1 did not show signs of hunger after dietary restriction. Also, the mutant animals were unable to maintain normal body temperature after fasting, unlike wild-type controls.

Conversely, overexpression of Sirt1 led to greater physical activity and responsiveness to ghrelin, a hormone that signals an empty stomach, than those in wild-type animals.

The team also pinpointed the particular hypothalamic neurons in which Sirt1 is likely to act and found that Sirt1 is needed for these cells to relay signals from ghrelin to a brain circuit that uses orexin, a hormone that promotes feeding.

"Sirt1 acts in a very particular area of the hypothalamus to regulate physical activity and thermal regulation," said Imai. "Sirt1 increases the levels of orexin receptors, making these neurons more sensitive to ghrelin."

\section{Restrictive behavior}

Both Coppari and Imai believe their studies make a good case for selectively activating hypothalamic SIRT1 to ramp-up energy-burning behavior as an antidote to a high-fat diet or as an aid to dieting.

"Activating SIRT1 in this area of the brain should mimic caloric restriction," said Imai.

The challenge is how to exclusively activate the right neurons. Both researchers noted that the metabolic functions of SIRT1 are extremely complex and that inappropriately activating the enzyme in some parts of the brain and periphery may have undesirable effects.

"In general, we don't think that activating SIRT1 everywhere is good," said Imai.

Coppari agreed: "Hitting every neuron in the brain would not be a good thing."

Coppari favors a brain cell-selective agonist of SIRT1 that takes advantage of the hormone receptor profile of the hypothalamus. For example, his team is exploring "a bait that's attached to the SIRT1 drug to deliver it only to certain hypothalamic neurons" such as those expressing the leptin receptor.

Imai favors activating SIRT1 using brain-permeating derivates of nicotinamide mononucleotide, a small molecule metabolite that regulates the enzyme's activity.

Imai's patents on the use of nicotinamide derivatives for metabolic indications are available for licensing. Imai is on the scientific advisory board of GlaxoSmithKline plc's Sirtris Pharmaceuticals Inc. unit, 
which is developing small molecule SIRT1 agonists for a range of indications. The company was not involved in Imai's study, however.

Coppari did not patent his findings.

\section{Big man on hippocampus}

Meanwhile, a team at the Massachusetts Institute of Technology found that SIRT1 may play a role in the hippocampus, the brain region most affected by Alzheimer's disease (AD). A group led by Leonard Guarente, professor of biology, looked at the effect of knocking out Sirt1 in a mouse model of AD. ${ }^{3}$

First, the researchers found that brain-specific disruption of Sirt1 led to higher levels of $\beta$-amyloid (A $\beta$ ) plaques, one of the hallmarks of the disease, than normal expression of Sirt1. The Sirt1 knockouts also had more severe behavioral defects and lower survival rates than regular $\mathrm{AD}$ mice.

Guarente believes that SIRT1 affects the expression of ADAM metallopeptidase with thrombospondin type 1 motif 10 (ADAM10), a protease that converts amyloid- $\beta$ (A4) precursor protein (APP) into a benign version that does not form plaques. Indeed, in mouse brain extracts, brain-specific Sirt1 knockouts had lower expression of and proteolytic activity by ADAM10 than wild-type mice.

Guarente noted that prior mouse genetic studies have implicated Sirt1 in other neurological disorders such as Parkinson's disease (PD) and Huntington's disease (HD). He said the enzyme likely has multiple functions and that the AD-specific effect on ADAM10 is one of many ways in which SIRT1 acts as a neuroprotective factor.

Results were reported in Cell and are covered by patents that are licensed to Sirtris, where Guarente also is a scientific advisory board member.

\section{SIRT1 with certitude}

The findings in the three new papers point to a more targeted approach to treating metabolic and neurodegenerative disease than activating SIRT1 everywhere. Although several companies have tried to find SIRT1 agonists, only Sirtris has thus far brought such compounds into the clinic. ${ }^{5}$

Sirtris's GSK2245840/SRT2104 and GSK184072 are in Phase II testing for type 2 diabetes. SRT501, a formulation of the natural compound resveratrol, in is Phase II testing for multiple myeloma (MM).

Sirtris CEO George Vlasuk told SciBX the company has a number of brain-penetrating SIRT1 agonists in preclinical development.

The impact of SIRT1 on feeding behavior and A $\beta$ production makes the enzyme "a very attractive mechanism for drug development. We now have compounds that can get into the brain and we're seeing effects in metabolic and neurodegenerative disease models," said Vlasuk.

"There's a tremendous premium on SIRT1 activators that are brain permeable," Guarente told SciBX. "Sirtris has some compounds that they think might get into the brain, and we plan on testing them."

Vlasuk said Coppari's metabolism findings are in line with work presented this year by Sirtris researchers at the American Diabetes Association meeting in Orlando.

"It's very nice to see in Coppari's paper that SIRT1 regulates leptin signaling," said Vlasuk. "We've looked at leptin receptor-deficient models and we know that leptin is involved in the response to our compounds."

Vlasuk noted that the hypothalamus is relatively accessible thanks to its role in sensing peripheral hormones. Thus, he said, it is possible that Sirtris' compounds in the clinic for metabolic indications could be acting in part by targeting SIRT1 in the hypothalamus.

Sirtris' new compounds can enter the brain but aren't excluded from the rest of the body, and Vlasuk said getting exclusively "brain-selective compounds will be tough."

Even if it is possible to agonize SIRT1 in the brain, it's unclear whether doing so would have a significant therapeutic effect.

Valter Longo, associate professor of biological sciences at the University of Southern California, said that overexpression of SIRT1 in many neurological and metabolic disease models has not shown much efficacy.

"While SIRT1 is essential for normal functions, there's no indication that increasing its activity would have any effect in disease," said Longo.

Last month, Longo and colleagues published a report on the role of hippocampal SIRT1 on learning and memory. ${ }^{6}$ In that study, deletion of Sirt 1 caused cognitive problems, but overexpressing the enzyme did not improve learning and memory beyond the level seen in genetically normal controls.

Vlasuk acknowledged that "the effects of Sirt1 overexpression are not as profound as the effect of knockouts," but he cited unpublished data generated by Sirtris that "suggest that upregulation of SIRT1 has some positive effects in disease settings."

Peter DiStefano, CSO of Elixir Pharmaceuticals Inc., noted that SIRT1 knockout mice "have a lot of deficits from birth," so it's best to focus on highly localized inactivation or overexpression of the enzyme to understand its function in the brain.

"If you can get a SIRT1 activator that's real, there's a good case for activating SIRT1" in the brain, DiStefano said. However, he noted, protein deacetylases like SIRT1 "have many substrates and are found in a lot of tissues, so you could have a specificity problem," he noted.

Elixir and Siena Biotech S.p.A. have SEN0014196 (EX-527), a SIRT1 antagonist, in Phase I testing to treat HD.

DiStefano suggested that identifying the specific proteins that are deacetylated by SIRT1 in the hypothalamus and hippocampus might help to identify alternative therapeutic targets with brain-specific effects.

Osherovich, L. SciBX 3(31); doi:10.1038/scibx.2010.941

Published online Aug. 12, 2010

\section{REFERENCES}

1. Ramadori, G. et al. Cell Metab.; published online July 4, 2010; doi:10.1016/j.cmet.2010.05.010

Contact: Roberto Coppari, The University of Texas Southwestern Medical Center at Dallas, Dallas, Texas e-mail: roberto.coppari@utsouthwestern.edu

2. Satoh, A. et al. J. Neurosci.; published online July 23, 2010; doi:10.1523/JNEUROSCI.1385-10.2010

Contact: Shin-ichiro Imai, Washington University in St. Louis School of Medicine, St. Louis, Mo.

e-mail: imaishin@wustl.edu

3. Donmez, G. et al. Cell; published online July 23, 2010;

doi:10.1016/j.cell.2010.06.020

Contact: Leonard Guarente, Massachusetts Institute of Technology, Cambridge, Mass.

e-mail: leng@mit.edu 


\section{ANALYSIS}

\section{TARGETS \& MECHANISMS}

4. Yu, J. \& Auwerx, J. Ann. NY Acad. Sci. 1173, E10-E19; published online Sept. 11, 2009; doi: 10.1111/j.1749-6632.2009.04952.x

5. Osherovich, L. BioCentury 16(20), A9-A10; May 8, 2008

6. Michán, S. et al. J. Neurosci. 30, 9695-9707 (2010)

COMPANIES AND INSTITUTIONS MENTIONED

American Diabetes Association, Alexandria, Va.

Elixir Pharmaceuticals Inc., Cambridge, Mass.
GlaxoSmithKline plc (LSE:GSK; NYSE:GSK), London, U.K. Massachusetts Institute of Technology, Cambridge, Mass. Siena Biotech S.p.A., Siena, Italy

Sirtris Pharmaceuticals Inc. (NASDAQ:SIRT), Cambridge, Mass.

University of Southern California, Los Angeles, Calif.

The University of Texas Southwestern Medical Center at Dallas, Dallas, Texas

Washington University in St. Louis, St. Louis, Mo. 